\title{
Eye movement analysis as a tool to evaluate virtual reality systems and human behaviour
}

\section{Análise do movimento ocular como ferramenta para avaliar sistemas de realidade virtual e comportamento humano}

Virtual Reality technologies are evolving at a fast pace. It is important to establish if these novel solutions met the goals they were developed for (i. e., to evaluate if they are effective). These technological-based products, whether based on a conventional PC setup or an immersive virtual reality setup, predominantly rely on the stimulation of the sight. With this, the eye movement analysis appears as an important metric to evaluate technological environments. Through eye movement analysis can establish how persons collect information and what influences their actions and decisions and determine certain preferences or behaviours.

The capture of eye movements can be achieved using eye-trackers, which can be categorized into three different categories:

- Conventional eye trackers generally consist of a pair of regular eyeglasses with two small infrared cameras mounted on the eyeglasses and pointed towards the eyes and one small camera in front of the eyes to capture the scene which the user is looking at. Then, the data captured by the infrared cameras is processed together with the data captured from the front camera, so it is possible to overlay the real scene footage with a representation of the spots of the regions that the user looked at.
- PC-based eye-trackers, are used in conventional PC environments. These eye-trackers consist of a bar with infrared cameras inside that can be coupled to the top or bottom of the display and oriented to the user's eyes. A software then post-processes the information to overlay the user's eye movements to the content that was being displayed.

- Virtual Reality eye-trackers are achieved by mounting infrared cameras pointed to the eyes right below the head-mounted displays' lenses. Then, similar to pc-based eye-trackers, post-processing overlays the displayed content and eye movements.

The major outcome of the eye-tracking devices is overlaying what the user is watching and his eye movements. To achieve this overlay with precision, first a calibration step at the beginning of the usage of the eye-tracker. In addition to this outcome, a set of visual maps and metrics need to be set up. Visual maps consist of a heat map of visual attention, focus map, and metrics of the number of saccades, the number of fixations, and their durations

The usage of eye-tracking techniques to study human behaviour was already addressed on PsychTech by two studies, 
namely the study entitled "Physical exercise, female preference in corporal dimensions in men: A comparative study on eye-tracking" (Medon, et al., 2020) and the study entitled "The Position Of The Target In The Influence Of Eye Movement On Navigational And Informative Research Tasks" (Vasconcelos-Raposo et al., 2017). Nevertheless, there are still many research gaps open that can be pursued in two perspectives: how the features of a virtual reality environment affect the user; and how different factors can be used to condiction behaviours within virtual environments. As so, works that make use of eye-tracking analysis are encouraged to be submitted to PyschTech.

\section{REFERENCES}

Medon, A., Gonçalves, L., Amorim, M., \& Coelho, H. (2020). Physical exercise, female preference in corporal dimensions in men: A comparative study on eye-tracking. PsychTech \& Health Journal, 4(1), 49-63. https://doi. org/10.26580/PTHJ.art28-2020

Vasconcelos-Raposo, J., Teixeira, C. M., Alves, C., Lopes, H., Mendes, M., Andrade, P., \& Melo, M. (2017). The Position Of The Target In The Influence Of Eye Movement On Navigational And Informative Research Tasks. PsychTech \& Health Journal, 1 (1), 14-20. https://dx.doi.org/10.26580/PTHJ.art22017 